AMAZONIA PERUANA - Vol.IV - N: 7 - Pág. 311-63

\title{
HACIA LA RECONSTRUCCION DE LOS PATRONES COMUNALES DE ASENTAMIENTO DURANTE LA PREHISTORIA DE LA CUENCA AMAZONICA*
}

\author{
Thomas P. Myers \\ Museo Estatal de la \\ Universidad de Nebraska.
}

In this article the author reviews the ethnohistorical and ethnographic information related to settlement patterns in Amazonia on the basis of the overall characteristics of Tropical Forest Culture. The archaeological evidence for difforent cultures at different phases and for different regions is analyzed with the diverse ethnographic settlement patterns.

An attempt is made to correlate this settlement patterns with levels of socio. political complexity as a way to reconstruct prehistoric social processes. Finally, The Betty Meggers and Clifford Evans theories on population movement and ipclo-political development of Amazonia are discussed.

Cet article reprend l'information ethno-historique ef ethnographique concornant les modèles de peuplement en Amazonie à partir des caractéristiques de la culture de la forêt tropicale. L'auteur analyse les données archéologiques des divers cultures dans ses différentes phases aussi comme dans ses propres régions pour les opposer tout de suite aux différente modèles ethnographiques do peuplement. L'auteur se propose d'etablir les correspondances entre les modeles et les niveaux de complexité socio-politiques comme principe pour la reconstruction dès procès sociaux pré-historiques. Finalment il met en question lo modèle de peuplement et de développement socio-politique dans l'Amazonie présenté par les Drs. B. Meggers et C. Evans.

In dem vorliegenden Artikel behandelt der Autor ethnohistorische und ethnographische Informationen in Bezug auf Siedlungsformen auf der Basis der allgemeinen Charakteristik der 'Tropical forest culture'. Die archäologische Evidenz zu verschiedenen Kulturen aus unterschiedlichen Zeithorizonten und verschiedenen geographischen Regionen wird ebenso analysiert, wie verschiedene ethnographische Siedlungsformen. Es wird der Versuch unternommen, diese Siedlungsformen mit verschiedenen Ebenen sozialer Komplexität zu korelieren, um einige prehistorische soziale Prozesse zu rekonstruieren. Schliesslich werden noch die B. Meggers und Cl. Evans-Theorien über Populationsbewegungen in Amazonien diskutiert.

* Publicado en inglés en VARIATIONS IN ANTHROPOLOGY: Essays in honor of John C. Me Gregor. Editado por Donald W. Lathrap y Jody Douglas. Urbana: Illinois Archacological Survey.

Traducción: Luciana Proaño. 
Tradicionalmente, nuestra civilización ha mirado la selva con una mezcla de temor y asombro, característica de un pueblo forzado a lidiar con un medio ambiente desconocido. Quizá este enfoque tenga su origen en las experiencias de las primeras expediciones españolas que atravesaron la cordillera peruana hacia la "Tierra de la Canela", donde buscaban riquezas pero encontraron la muerte $y$ el desastre. Los portugueses parecen haber tenido una experiencia bastante distinta con el bosque tropical, quizá por las expectativas que de él tenían. Claro que uno podría fraguar como argumento que nuestra relación histórica fue mayor con las experiencias españolas que con las portuguesas debido a los contactos que los piratas ingleses tenían con el comercio español del oro y con sus puertos en Panamá y Colombia. Pero parece mejor acertado examinar más de cerca los dos últimos siglos de contacto para hallar el germen de las ideas que influyen en nuestro pensamiento acfual.

Los finales del siglo dieciocho y comienzos del diecinueve vieron florecer el interés cientifico europeo en el mundo. Este era, en parte, una reflexión acerca de la expansión del imperio, especialmente del Imperio Británico, que estaba en curso justamente en ese período. Los viajes del capitán Cook tuvieron un interés tanto científico como político; casi un siglo más tarde, en un viaje similar de exploración, viajaba un joven naturalista: Charles Darwin. Simultáneamente otros naturalistas penetraban la selva del Amazonas: Spruce, Wallace y Bates se suentan entre los más conocidos. Al tiempo que observaban la flora y la fauna, estos hombres observaron también a los nativos con quienes trabajaban. Por ejemplo, Bates comentaba que los indígenas tenian un "temperamento flemático, apático; frialdad de deseo y falta de sentimiento, poca curiosidad y un intelecio lento". Añadía que "su imaginación es de una calidad insulsa, oscura y parecía que las emociones nunca los movian: amor, pena, admiración, miedo, sorpresa, alegría, entusiasmo. Estas son las características de toda la raza". (Bates, 1864: 293). En fecha algo posterior, durante un viaje a Guyana, Im Thurn observa que "la vida de los indios se compone de estallidos alternados de energía e inactividad relativa". Pero prosigue que "esta inactividad y descuido del tiempo no se debe a ninguna pereza digna de culpa" sino más bien a que las herramientas anteriormente manufacturadas por los indigenas con gran esfuerzo, habian sido reemplazadas por bienes europeos que se podían conseguir fácilmente a través del intercambio. Concluye que "acorde al viejo y certero principio que el trabajo es bueno para el hombre, probablemente este hecho también explica en gran medida la tan común degeneración de los salvajes en presencia de la civilización". (1883: 269-270). Esta aguda observación final es rara entre los viajeros del siglo diecinueve y su significación sigue siendo despreciada por quienes nos agrada calificar de observadores científicos modernos.

\section{UNA CARACTERISTICA DE LA CULTURA DE BOSQUE TROPICAL}

Indudablemente, el Volumen 3 del Handbook of South American Indians es el único tratamiento de los pueblos de Bosque Tropical, de gran influencia. Caracteriza a la región y la cultura diferenciándolas de regiones y culturas adyacentes. Según Lowie el complejo del Bosque Tropical se distingue de las 
civilizaclones andinas más altas por su falta de refinamiento arquitectónico y matalúrgico, siendo más desarrollada que las culturas con la economía de caza-recolección de los Botocudo y con la mediana horticultura de los Apinayé. In esencia, la cultura incluye el cultivo de tubérculos, especialmente yuca amarga, eficientes embarcaciones para rio, el uso de hamacas a manera de camas, y la manufactura de cerámica (1948: 1). Más aún, Lowie cree que las aldeas orlginales no eran características del Bosque Trapical en su totalidad, aunque reconoce algunas excepciones, por lo menos una de las cuales puede haberse debido a la influencia de las misiones (1948: 16-17). La influencia política genoralmente está restringida a una cabeza pese a que se decía que los Yurunas tenían jefes supremos (Lowie, 1948: 32).

In general, la exposición de Lowie era una justa caracterización de las Hilbus de Bosque Tropical, existentes cuando él escribió. 'Sus fuentes tienen una fecha modal de publicación alrededor de 1925, por lo que répresenta la altuación tal como se daba durante el primer cuarto del siglo XX. Evidentemante Lowie trató de resumir la condición de la Cultura de Bosque Tropical en esa época más que realizar una caracterización histórica de ella. Sin ambargo, es curiosa su selección de rasgos principales porque incluye por un lado, técnicas adaptativas tan básicas como el cultivo de tubéreulos y el uso de eficientes embarcaciones de rlo $y$, por otro, la manufactura de cerámica que es general e importante pero no esencial ni contribuye por sí misma a un diagnóstico. En este listado de rasgos fundamentales también se incluye - l uso de hamacas para dormir, criterio que es de un orden totalmente dlotinto y simplemente no pertenece a una lista de rasgos principales.

En su artículo interpretativo de las tribus de "montaña" Steward señala que "la comunidad aborigen de 'montaña' consistió típicamente de una a varias familias -15 a 30 personas - cada una viviendo en una pequeña casa... las cuales estaban dispersas en intervalos a lo largo del curso de las aguas... o aisladas en el monte para protegerse de la guerra y las correrías de esclavos. Sin embargo, unas euantas tribus gozaban de comunidades mucho más grandes: las aldeas Tupí contaban con varios cientos de personas; en 1962 los asentamientos Cayuvava promediaban 540 por aldea, las comunidades Mostene promediaban 166 en 1682. Estos tamaños parecen wer auténticos pero no es seguro si dependen de mayores recursos locales y una población excepcionalmente densa o de un sentido político más desarrollado..." (Steward, 1948a: 527). Evidentemente, Steward cancebía los asentamientos de montaña como pequeños centros aislados en los bosques, las comunidades más grandes eran excepciones que podían explicarse según sus condiciones lacales particulares. De esta manera podía señalar que "en el período histórico se dio una tendencia hacia el crecimiento de la comunidad, pese a que es interesante notar que las aldeas de las misiones con varios

I: El tormino "montaña" aparece siempre en castellano en el texto original. - 
cientos a miles de habitantes se desintegraron al cierre del periodo misional cuando los pueblos tendieron a reasumir su separatismo nativo". (1948a: 527). Continúa que la "autoridad política se centraba en la cabeza familiar que controlaba los viajes, el quehacer guerrero y la limpieza de la chacra. Liderazgos de mayor envergadura se daban sólo durante alianzas de guerra temporales y en tiempos muy recientes cuando parece claro ser una institución impuesta por el hombre blanco". (1948a: 528).

En su caracterización resumida de las áreas culfurales del Bosque Tropical como un todo, Steward observa que "las culturas básicas del Bosque Tropica! aparecen principalmente en las áreas asequibles a rutas de agua, tanto la costa como los ríos grandes, mientras que las tribus más simples o marginales fienden a estar distribuidas en una gran " $U$ " en la periferia de la Cuenca Amazónica. Significativamente, esta " $U$ " que incluye la hoya Amazonas-Orinoco, la ladera oriental de los Andes, partes del Mato Grosso y parie de la cordillera del Este brasileño, posee la mayor cantidad de indígenas no aculfurados. El monte es remoto y los arroyos pequeños, haciendo a la zona de difícil acceso para los pueblos esencialmente ribereños tanto en tiempos precolombinos como en la actualidad. Claramente se infiere que lo que se conoce como la típica cultura de Bosque Tropical "...corrió a lo largo de la costa y subió por los ríos principales llegando hasta donde los arroyos son menos navegables, dejando a las tribus del inferior en un nivel más primitivo... Por otro lado, con mayor frecuencia que los pueblos desarrollados del Bosque Tropical, los marginales tienen sibs, mitades y otras elaboraciones sociales". (Steward 1948a: 883-885). A partir de los datos de distribución añade que la "culfura de Bosque Tropical se ex́pandió hacia el sur por la costa del Atlántico hasta el Amazonas y por los afluentes de éste especialmente río arriba... Se postula a las Guyanas y al bajo Amazonas como centro de dispersión ante la evidencia de la probable dirección del movimiento cultural en el Bosque Tropical y de la riqueza de restos arqueológicos en aquellos centros... La fuente última de la Culfura de Bosque Tropical puede buscarse hasta eh el área Circum-Caribeña". (Steward, 1948b: 886). En contraste, señalaba que pese a que algunas tribus ribereñas tenian aldeas grandes y numerosas no existía un registro de la conformidad política y económica de una población densa.

Como resumen de lo que fue la cultura de Bosque Tropical a fines del siglo XIX y comienzos del XX, sería dificil mejorar las relaciones presentadas por Lowie y Steward; pero ni Lowie ni Steward parecen haber tomado en cuenta acertadamente el sentido de la diferencia entre los relatos de los pueblos del Bosque Tropical en los siglos XVI y XVII, con los cuales por to menos Steward estaba familiarizado, y aquellos de fines del XIX y comienzos del XX. Quizás porque simplemente creían que las fuentes tempranas no eran confiables porque habian sido escritas por aventureros cegados por la codicia del oro, 0 , por misioneros que sacaban ventaja de sus desmesuradas expectaivas. Como se verá más adelante, el análisis de estas fuentes iniciales conlleva una imagen bastante diferente a la presentada por observadores posteriores. Yendo un poco más lejos, se abre a cuestionamiento la significación de la dicotomía. entre las eulturas Circum-Caribes del período de confacto y las culturas de 
Hesque Tropical de nuestros tiempos. Steward supone que existe una distinción Ilpológica entre ambas y que una deriva de la otra. Debemos coincidir con el primer punto, con el segundo no podemos. Una distinción tipológica del mismo -ntilo se da entre las culturas de bosque tropical de los siglos XVI y XVII y los indígenas actuales. Una existe y la otra ya no. Pongámoslo más almple, Steward está comparando las culturas Circum-Caribes de la época del Contacto con las culturas del Bosque Tropical que han sufrido casi cuatro siglos de cambios culturales forzados. Casi no podemos suponerlas similares.

Steward habla identificado un probable origen de la cultura de Bosque Tropical, $y$ al hacerlo formuló un problema que podía ser resuelto a través de lécnicas arqueológicas. Antes que el volumen estuviera siquiera en prensa, Evans y Meggers ya estaban haciendo planes para verificar las hipótesis. En -l último informe sobre su investigación en la boca del Amazonas resumen la Cultura de Bosque Tropical en base a la información contenida en el Handbook. Incluso formalizan la Cultura de Bosque Tropical como un Nivel de Desarrollo Cultural; el Nivel Marginal y el Nivel Circum-Caribe, (Meggers y Evans, 1957: 18). Las características de cada nivel aparecen simplificadas en la Tabla 1.

Tabla 1. Resumen de las Características de Tres Niveles de Cultura Nativa Sudamericana.*

$\begin{array}{llll}\text { Característica } & \text { Marginal } & \begin{array}{l}\text { Bosque } \\ \text { Tropical }\end{array} & \text { Circum-Cari } \\ \text { agricultura } & \begin{array}{l}\text { mótil } \\ \text { ausente }\end{array} & \text { mótil } & \text { permanente } \\ \begin{array}{l}\text { uso de recursos } \\ \text { silvestres }\end{array} & \text { extensivo } & \text { importante } & \text { infrecuente } \\ \begin{array}{l}\text { concentración } \\ \text { de población }\end{array} & \text { poca } & \begin{array}{l}\text { poca a } \\ \text { regular }\end{array} & \text { mucha } \\ \begin{array}{l}\text { división del } \\ \text { trabajo }\end{array} & \begin{array}{l}\text { sin } \\ \text { clases }\end{array} & \begin{array}{l}\text { sin } \\ \text { clases }\end{array} & \text { en clases } \\ \text { control } & \text { difuso } & \text { difuso } & \text { formal }\end{array}$

* A partir de Meggers y Evans, 1957: 18. 
Luego de haber resumido los datos etnográficos sobre las tribus de Bosque Tropical presentes en el Handbook, Meggers y Evans retoman los "indicadores básicas del patrón de Bosque Tropical" de Lowie: "agriculiura, embarcaciones, hamacas y cerámica" (de nuevo, èpor qué hamacas?) "sólo queda la cerámica para el arqueb́logo... es el único vínculo existente entre el pasado arqueológico y el presente etnográfico". (Meggers y Evans, 1957: 24). Pero añaden que de la distribución de la cerámica en el asentamiento arqueológico se puede deducir la extensión y composición del lugar. Estos datos pueden com. pararse a los que se tienen sobre los pueblos existentes y "cuando la corres. pondencia sea positiva, puede asumirse con bastante confianza que los aspecfos sociapolíticos, religiosos y de materiales perecederos de la culfura también coincidirán en rasgos generales". (Meggers y Evans, 1957; 25). (a)

Resumiendo estas múltiples versiones acerca de la Cultura de Bosque Tro. pical, surge un esquema general. La esencia de la Cultura de Bosque Tropical es el cultivo de fubérculos y el transporte acuático eficiente. Si se sigue a Lowie la cerámica y las hamacas también son restos importantes. Los asenta. mientos son pequeños (lo normal sería un máximo de 50 a 100 personas, quizás organizadas en líneas de parentesco bilaterales) y no permanentes, durando no más de unos cuantos años -el tiempo que tome agofar los campos aledaños. El liderazgo político estaría en manos de un hombre en virtud de su edad, habilidad o quizás sus vínculos de parentesco, pero sin ningún poder de mando. Con seguridad no existía ninguna unidad política supra-local excepto bajo circunstancias muy inusuales y de poca duración y mucho menos nada que se parezca a un estado. La religión estaba poco elaborada, como podría esperarse de pueblos tan simples, faltando todo lo que integra el culto de idolos y templos que caracterizaba a las tribus Circum-Caribes (Steward y Faron, 1959: 286).

\section{LA EVIDENCIA ARQUEOLOGICA}

Desde 1957 ha surgido buena cantidad de evidencia acerca de la pre-historia de la Amazonía, aunque aún poco abundantes. Los datos se encuentran dispersos geográficamente desde la boca del Amazonas, pasando por el Amazonas central, hasta el Amazonas peruano y río arriba de un afluente principal al norte: el rio Napo, y de un afluente principal del sur: el río Ucayali. Desafortunadamente, a pesar del defalle de los informes finales, ninguno de estos trabajos puede considerarse más que una excavación de prueba según los standards norteamericanos. Un hueco de prueba en un lugar, dos en otro, es la característica de la mayar parte del trabajo realizado hasta el momento. El trabajo en el Ucayali Central ha sido un poco más defallado, pero en UCA 6 es donde se han realizado las excavaciones más extensas, se ha excavado un poco más de 1,000 pies cuadrados, mientras que en UCA 34 -el siguiente lugar más investigado-, sólo se ha abierto 720 pies cuadrados en un asentamiento enorme cuya dimensión soblo la sospechamos vagamente. Al momento de escribir este artículo se llevan a cabo otras investigaciones en muchas zonas de la Cuenca Amazónica por lo que pronto tendremos más información. 
Tabla 2. Resumen del tamaño y dimensión de los asentamientos de la isla Marajó."

\begin{tabular}{|c|c|c|c|c|}
\hline Acentamiento & Faso & Caracteristica & 'Dimensiones: & Area \\
\hline \multirow{2}{*}{$1-7$} & Ananatuba & pequeña elevación & $30 \times 30 \mathrm{~m}$ & $707 \mathrm{~m}^{2}$ \\
\hline & Ananatuba & pequeña elevación & $35 \times 22(?)$ & $770^{*}$ \\
\hline $1-8$ & Ananatuba & pequeña elevación & $30 \times 30$ & 707 \\
\hline $1-9$ & Ananatuba & pequeña elevación & $20 \times 20$ & 314 \\
\hline $1-10$ & Ananatuba & túmulo bajo & $50 \times 10$ & 500 \\
\hline$i-26$ & Ananatuba & isla & $85 \times 85$ & 5677 \\
\hline \multirow[t]{3}{*}{$i-5$} & Mangueiras & túmulo 1 & $55 \times 34$ & 1870 \\
\hline & Mangueiras & fủmulo 2 & $52 \times 30$ & 1560 \\
\hline & Mangueiras & túmulo 3 & $25 \times 25$ & 491 \\
\hline \multirow{2}{*}{$i-7$} & Mangueiras & pequeña elevación & $30 \times 30$ & 707 \\
\hline & Mangueiras & pequeña elevación & $35 \times 22(?)$ & $770 *$ \\
\hline$i-13$ & Mangueiras & pequeña elevación & $75 \times 30$ & 2250 \\
\hline \multirow[t]{2}{*}{$i-16$} & Mangueiras & túmulo 1 & $70 \times 70$ & 3850 \\
\hline & Mangueiras & túmulo 2 & $70 \times 15$ & 1050 \\
\hline$i-17$ & Mangueiras. & banco elevado & $150 \times 50$ & 7500 \\
\hline \multirow[t]{6}{*}{$i-4$} & Formiga & †úmulo 1 & $100 \times 20$ & 2000 \\
\hline & Formiga & †úmulo 2 & $80(?) \times 20$ & 1600 \\
\hline & Formiga & túmulo 3 & $3.5 \times 8$ & 280 \\
\hline & Formiga & túmulo 4 & $35 \times 8$ & 280 \\
\hline & Formiga & †úmulo 5 & $25 \times 5$ & 125 \\
\hline & Formiga & túmulo 6 & $25 \times 5$ & 125 \\
\hline \multirow[t]{3}{*}{$i-6$} & Formiga & túmulo 1 & $60 \times 8$ & 480 \\
\hline & Formiga & túmula 2 & $10 \times 10$ & 78 \\
\hline & Formiga & túmulo 3 & $18 \times 18$ & 254 \\
\hline$i-18$ & Formiga & túmulo & $26 \times 6$ & 150 \\
\hline$i-30$ & Formiga & túmulo & $47 \times 23$ & 1081 \\
\hline$j-33$ & Formiga & túmulo & $95 \times 46$ & 4370 \\
\hline $1-14$ & Marajoara & túmulo I & $121 \times 56$ & 6776 \\
\hline
\end{tabular}




\begin{tabular}{|c|c|c|c|c|}
\hline Asentamiento & Fase & Caracteristica & Dimensiones & Area \\
\hline & Marajoara & túmulo & $85 \times 45$ & 3825 \\
\hline & Marajoara & túmulo 3 & $75 \times 65$ & 4875 \\
\hline$i-15$ & Marajoara & túmulo & $255 \times 30$ & 7650 \\
\hline & Marajoara & túmulo 2 & $5 \times 1 / 2$ & 2.5 \\
\hline & Marajoara & túmulo 3 & $32 \times 8$ & 256 \\
\hline & Marajoara & túmulo & $100 \times 6$ & 600 \\
\hline , & Marajoara & túmulo. & $45 \times 8$ & 360 \\
\hline & Marajoara & túmuio 6 & $50 \times 8$ & 400 \\
\hline & Marajoara & túmulo & $60 \times 15$ & 900 \\
\hline & Marajoara & túmulo 8 & $45 \times 11$ & 495 \\
\hline & Marajoara & túmulo 9 & $20 \times 10$ & 200 \\
\hline & Marajoara & túmulo 10 & $30 \times 15$ & 450 \\
\hline & Marajoara & túmulo 11 & $18 \times 14$ & 252 \\
\hline & Marajoara & túmulo 12 & $12 \times 12$ & 113 \\
\hline & Marajoara & túmulo 13 & $18 \times 10$ & 180 \\
\hline & Marajoara & fúmulo 14 & $51 \times 30$ & 1530 \\
\hline & Marajoara & túmụlo 15 & $30 \times 11$ & 330 \\
\hline & Marajoara & fúmulo 16 & $140 \times 16$ & 2240 \\
\hline & Marajoara & túmulo 17 & $250 \times 59$ & 14750 \\
\hline & Maialoara & túmulo 18 & $68 \times 68$ & 3633 \\
\hline & Maraloara & fúmulo 19 & $25 \times 25$ & 491 \\
\hline & Murajocara & túmuilo 20 & $5 \times 2$ & 10 \\
\hline 107 & Marajoara & túmulo & $140 \times 40$ & 5600 \\
\hline 104 & Marejoura & túmulo & $60 \times 25$ & 1500 \\
\hline 113 & Arine & banco elevado & $100 \times 50$ & 5000 \\
\hline 111 & Aros & terrono elevado & muy peq. & $\ldots$ \\
\hline
\end{tabular}


Tabla 3. Resumen de los Tamaños y Dimensiones de los Asentamientos en Terriforio Amapá."

A - 5

Aruá

túmulo

$10 \times 10 \mathrm{~m}$

$78 \mathrm{~m}^{2}$

$\begin{array}{ll}\text { A-1 Maragao equeña } & \text { elevación }\end{array}$

A-2

Mazagao

banco alto

$110 \times 60$

6600

A-3

Mazagao

banco alto

$83 \times 52$

4316

A-4

Mazagao

colina alta

$30 \times 30$

706

$25 \times 25$

156

(cumbre

de colina)

Mazagao colina alta

$5 \times 4$

(zona

funeraria)

A-5

Mazagao

fúmulo

$10 \times 10$

A-6

Mazagao

colina

$83 \times 75$

6225

A-9.

Aristé

poqueña

elevación

$100 \times 100$

7857

A-12

Aristé

banco alto

$100 \times 100$

7857

* Tomado de Meggers y Evans, 1957. 
Tabla 4. Resumen de los Tamaños y Dimensiones de los Asentamientos Habitacionales de las Islas Moxiana y Caviana.*

\begin{tabular}{|c|c|c|c|c|}
\hline$c-3$ & Mangueiras & $\begin{array}{l}\text { pequeña } \\
\text { elevación }\end{array}$ & $25 \times 25 \mathrm{~m}$ & $491 \mathrm{~m}^{2}$ \\
\hline$M-3$ & Acauan & $\begin{array}{l}\text { pequeña } \\
\text { elevación }\end{array}$ & $350 \times 15$ & 5250 \\
\hline$M-2$ & Aruá & banco & $15 \times 15$ (?) & 176 \\
\hline$M-7$ & Aruá & banco & $15 \times 15$ (?) & 176 \\
\hline$C-5$ & Aruá & $\begin{array}{l}\text { pequeña } \\
\text { elevación }\end{array}$ & $12 \times 12$ & 113 \\
\hline$c-6$ & Aruá & $\begin{array}{l}\text { pequeña } \\
\text { elevación }\end{array}$ & $75 \times 15$ & 1125 \\
\hline$C-7$ & Aruá & colina & $20 \times 7$ & 140 \\
\hline$C-8$ & Aruá & banco & $20 \times 8$ & 160 \\
\hline$C-10$ & Aruá & $\begin{array}{l}\text { pequeña } \\
\text { elevación. }\end{array}$ & $75 \times 20$ & 1500 \\
\hline$C-13$ & Aruá & colina & $30 \times 10$ & 300 \\
\hline$C-14$ & Aruá & banco alto & $15 \times 10$ & 150 \\
\hline$C-15$ & Aruá & banco & $5 \times 5$ & 19 \\
\hline
\end{tabular}

* Tomado de Meggers y Evans, 1957. 


\section{La boca del Amazonas}

Un análisis del récord arqueológico de los asentamientos en la boca del Amazonas (Meggers y Evans, 1957; Simões, 1967, 1969) resumidos en las Tablas 2, 3 y 4 revela varios giros en el desarrollo de los patrones de asentamiento y comunidad. En el período arqueológico temprano, los pueblos de la Fase Ananatuba elegian lugares en tierras altas naturales del interior de la isla, donde dejaron basurales típicamente de forma circular $u$ ovoide que cubrian poco más de media hectárea de terreno. Tales asentamientos siguieron siendo regla durante la Fase Mangueira, pero la dimensión máxima aumentó hasta aproximadamente tres cuartos de hectáreas de acumulación de deshecho de casi un metro de profundidad. Hay un cambio a asentamientos claramente lineales en la Fase Formiga (Tabla 5) durante la cual la gente seguía viviendo en acumulaciones de basura pero también se empezó a construir fúmulos ha. bitacionales. El área máxima de superficie de los asentamientos de la Fase Formiga es alrededor de media hecłárea, un poco menor que el asentamiento más grande de la Fase anterior, pero la población pudo haber sido la misma. En la Fase Marajoara encontramos un patrón de comunidad y asentamiento radicalmente distinto, numerosos túmulos lineales de dos anchos modales (Tabla 6) construidos a lo largo de un arroyo pequeño. El área máxima de superficie de un asentamiento alcanzó hasta las tres hectáreas y media.

Tabla 5. Razones de Ancho por Largo de los Asentamientos y Túmulos de la Boca del Amazonas.*
ancho: largo $1: 1$
$2: 1 \quad 3: 1$
$4: 1$
$5: 1$
$6: 1$
$7: 1$

Fase

\begin{tabular}{lccccccc}
$\begin{array}{l}\text { Ananatuba } \\
\text { Mangueriras }\end{array}$ & 4 & 1 & - & - & 1 & - \\
Formiga & 4 & 4 & - & 1 & - & - \\
Marajoara & 2 & - & - & 4 & 3 & - & 1 \\
Aruá & 5 & 6 & 1 & 3 & 1 & 1 & 4 \\
Mazagao & 2 & 2 & 3 & 1 & 1 & - & - \\
Aristé & 4 & 2 & - & - & - & - \\
Acauan & 2 & - & - & - & - & - \\
\hline
\end{tabular}

* Tomado de Meggers y Evans, 1957. 
En suma, se puede observar los siguientes cambios en la pre-historia Marajó: 1) El cambio de asentamientos ovalados a lineales, 2) El cambio de terrenos - levados nafurales a túmulos de acumulación de basura, a túmulos construidos artificialmente, y nuevamente a terrenos altos naturales, 3) El cambio del tamaño general de los asentamientos, de alrededor de media hectárea a tres hectáreas y media en la Fase Marajoara y nuevamente a una hectárea y media en la Fase Aruá, 4) El traslado desde el interior hacia los bancos de los ríos pequeños. La fase Formiga, parece haber sido una fase clave en el desarrollo cultural ya que es cuando se empieza a construir los fúmulos lineales artificiales. Esta próctica se continúa y se hizo más elaborada en la Fase Marajoara, largamente reconocida como una de las cumbres en la historia de la cultura amazónica. Hacia la Fase Protohistórica Aruá, la cultura alta había sido destruida en Marajó, probablemente debido a la introducción de la cultura y las enfermedades europeas.

Tabla 6. Ancho Absoluto de los Asentamientos lineales y Túmulos en la Boca del Amazonas.*

ancho en metros:

$\begin{array}{rrrrrrrrrrr} & 6 & 11 & 16 & 21 & 26 & 31 & 36 & 41 & 46 & 51 \\ 5 & 15 & 15 & 20 & 25 & 30 & 35 & 40 & 45 & 50 & 55\end{array}$

Fase

Formiga

Marajoara

$25-3$

$26 \cdot 6111112-1-3$

Aruá

Acauan

* Tomado de Meggers y Evans, 1957.

Sólo en el caso de la Fase Marajoara se ha sugerido una función diferencial del asentamiento. Meggers y Evans (1957) designaron algunos túmulos como destinados especialmente a propósitos funerarios, y Simões (1967) atribuye esta función a nada menos que siete de los diez túmulos que estaba investigando. Tal como reconocen los autores, esta especialización de funciones está bastante fuera de lugar en la Cultura de Bosque Tropical, pero su identificación permanece más como simple afirmación que como hecho establecido por excavaciones detalladas. Pese a que la Fase Aruá está ciertamente caraclerizada por cementerios de entierros en jarrones, la práctica bien puede haber sido producto de la influencia europea; mientras que los entierros en jarrones al interior de los asentamientos habitacionales es bastante característico de otras culturas del Bosque Tropical. De todas maneras, yo sospecho que otros 
túmulos habitacionales de la fase Marajoara también contienen entierros y que los túmulos funerarios contienen a la vez restos habitacionales. La situación se presta a la prueba arqueológica, que de una u otra forma, tendría poca ingerencia sobre el argumento principal ya que el hecho de existir una cultura relativamente avanzada en la Fase Marajoara no depende de la función diferencial del asentamiento, sino del tamaño de éste y de la complejidad de la cerámica. Si se tuviera que aplicar una prueba, estaría particularmente interesado en el Túmulo 18 en J-15 que es el único túmulo circular grande del lugar. El lugar J-6 de la Fase Formiga también sería interesante ya que sostiene igualmente un túmulo circular grande.

En todo caso es claro que algo especial sucedió durante la Fase Marajoara. No sólo llegó la cerámica a la cumbre local en variedad y perfección de producción, sino que también la construcción de grandes túmulos artificiales es indicativa de una sociedad en la cual existió algo más que la particularidad perezosa, característica de la Cultura de Bosque Tropical.

\section{El Amazonas Central}

Es on extremo difícil discernir un patrón convincente para los asentamientos del Amazonas central ya que muy pocos han sido identificados y muchos de ellos parecen contener componentes de más de una tradición cerámica. Este hecho es significativo por sf mismo puesto que atestigua la estabilidad geomorfológica de la región en el mismo sentido que los datos de radiocarbono de Sternberg sobre las islas en el río grande (Sternberg 1960). Al igual que los asentamientos Marajó, los del Amazonas central casi siempre están localizados en terrenos elevados, pero en vista de que no existe construcción de túmulos en esta región, las dimensiones de los asentamientos son práctica. mente las máximas. La inexactitud para establecer el tamaño de los asentamientos deriva del hecho de que los basurales sálo aparecen cuando el río ha penetrado el banco, destruyendo asf parte del asentamiento, o donde la utilización contemporánéa ha expuesto una porción del asentamiento arqueológico en una aldea o campo de roce y quema. En el monte solamente los árboles caídos o los huecos de pruaba, pueden demostrar si el terreno estuvo ocupado alguna vez. Incluso con estas dificultades es evidente que muchos asentamientos del Amazonas central eran considerablemente mayores que los de Marajó (comparar Tablas 2 y 7). Igualmente, los asentamientos del bajo Tapajós (Palmatary) parecen ser más grandes que los de la Fase Marajoara.

En la Tabla 7, he resumido la información que Hilbert da acerca de los asentamientos del Amazonas central. La interpretación de Hilbert sobre los restos cerámicos ha sido revisada por Lathrap (1970) quien encuentra elementos de hasta tres tradiciones cerámicas en una de las fases que menciona Hilbert. Sin embargo, esto necesariamente no significa que han habido tres ocupaciones distintas del asentamiento. Los datos no son suficientes para probar una hipótesis de ese tipo y tampoco Lathrap lo interpreta en ese sentido. Más bien, sugiere que el material cerámico en ciertos asentamientos es tran- 
Tabla 7. Resumen de los Tamaños y Dimensiones de Asentamientos en Amazonas Central.*

Asentamiento

Componentes

Características

Dimensiones

Area

Ponta do

Javarí

Horizontes

- de Reborde

Incisivo

Paradao

Divinópolis

Coari II

Caímbe

Horizonte Polieromo

Manacapurú

Refinaria

Tefé

Sao Joaquim

Pirapitinga
Jauarí.

Paradao

Paradao

Paradao

Caímbe
2 túmulos de conchas y 3 áreas de basura

tiestos:

$100 \times 45 \mathrm{~m}$

piedras:

$80 \times 30$

$4500 \mathrm{~m}^{2}$

2400 
sicional entre una tradición y otra. En otras palabras, las tradiciones cerámicas no son hechos aislados inmutables en todos los lugares y épocas sino simples recursos heurísticos con los cuales resumir nuestra comprensión de segmentos particulares de información. Como instrumentos deben abandonarse cuando dejen de servir a su función.

La subtradición Miracanguera de la Tradición Polícroma se puede identificar en tres asentamientos pero sólo se conoce la dimensión de dos de ellos, liacoatiara I y ll. Ambos asentamientos son tres y media a cuatro veces más largos que anchos. Pero al examinar el mapa de Hilbert (Hilbert, 1968: Karte 10) es posible que fueran simples rezagos de un asentamiento mucho más grande que se extendía anteriormente alrededor de una curva del río. Siendo imposible asegurar las dimensiones originales del asentamiento, es probable que haya sido mucho más largo que ancho, igual que los fragmentos que ha dejado.

La subtradición Guarita de la Tradición Policroma se encuentra en seis asentamientos, en tres casos asociada a elementos de la Tradición Barrancoide. Al examinar la estratigrafía en estos asentamientos Lathrap sugiere que la subtradición Guarita se deriva de la Tradición Barrancoide (Lathrap, 1968: 157). De estos tres sólo se conoce las dimensiones del asentamiento Manacapurú el cual es cinco veces más largo que ancho, cubriendo completamente 80 hectáreas. Los otros dos asentamientos de la Subtradición Guarita cuya dimensión se conoce son casi circulares, coincidiendo con la forma de la colina en que están ubicados. Estos cubren un área poco mayor a diez hectáreas y un poco menor a tres hectáreas respectivamente.

Los asentamientos de la Tradición de Línea Fina Incisiva son aproximadamente dos veces más largos que anchos, cubriendo sólo un poco más de una hectárea. Por lo menos en esta área, parecen ser mucho más pequeños que los asentamientos de la Tradición Policroma, pero en la boca del Tapajós (Palmatary, 1960) el tamaño de los asentamientos de la Tradición de Línea Fina Incisiva (Santarem) parece estar más cercano a los de la Tradición Policroma reportados por Hilbert.

En suma. parece que los asentamientos de la Tradición Policroma pueden estar localizados tanto en colinas como en diques antiguos. En el primer caso los asentamientos son, a grandes rasgos, circulares, según la forma de la colina. En el segundo, son claramente lineales, también según la forma de la elevación de terreno (Tabla 8). En cualquier instancia, son mucho más grandes que los asentamientos de la Tradición de Línea Fina Incisiva de la misma zona.

Igual que los asentamientos del Amazonas brasileño, los de Cushillococha - un lago cercano a la frontera entre Perú y Brasil (Harris, 1967)-tienden a ser mucho mayores que los de la boca del Amazonas. En los asentamientos AMA I y AMA 4 se determinó que los restos del Complejo Yanayacu, típico de la Tradición Polícroma, cubrian las del Complejo Nata que es difícil de vincular a las mayores unidades culturales hasta ahora descritas para la Cuenca Amazónica. AMA 1 es un asentamiento claramente lineal que cubre 
poco menos de seis hectáreas; pero AMA 4 es circular con una extensión de casi soblo un décimo de hectárea. La cerámica del complejo Cushillococha -qua no ha sido identificada con ninguna tradición cerámica exterisa del Amazonas, ni ubicada en el tiempo- también se halló en un asentamiento lineal de má - menos siete hectáreas. Un moderno asentamiento Tícuna era circular y ocu. paba alrededor de media hectárea de terreno.

Tabla 8. Resumen de los Tamaños y Dimensiones de los Asentamientos on Cushillococha.*

$\begin{array}{lllll}\text { AMA } 1 & \begin{array}{l}\text { Yanayacu } \\ \text { Nata' }\end{array} & \begin{array}{l}\text { Terraza } \\ \text { natural }\end{array} & 600 \times 100 \text { yard. } 60000 \mathrm{yds.2} \\ \text { AMA 2,5 } & \begin{array}{l}\text { Cushillococha } \\ \text { Nata }\end{array} & \begin{array}{l}\text { Terraza } \\ \text { natural }\end{array} & 700 \times 100 & 70000 \\ \text { AMA 3 } & \begin{array}{l}\text { Ticuna } \\ \text { AMA 4 }\end{array} & \begin{array}{l}\text { Terraza } \\ \text { natural }\end{array} & 80 \times 80 & 5028 \\ \text { Yanayacu } & \begin{array}{l}\text { Terraza } \\ \text { natural }\end{array} & 40 \times 40 & 1257\end{array}$

* A partir de Harris, 1967.

Mientras que sólo se pueden establecer algunas conclusiones generales acerca de los patrones comunales del área de Cushillococha, se demuestra una vez más la presencia de grandes asentamientos lineales. Uno de estos perfenece a la Tradición Polícroma, reforzando asi la identificación de los asentamientos lineales con esta tradición. El asentamiento circular identificado con la. Tradición Polícroma hallado en Cushillococha es tan pequeño que debería considerarse como satélite del mayor. En Cushillococha también vemos que. los asentamientos lineales no están asociados únicamente a la Tradición Policroma; como se verá más adelante, esta misma observación puede aplicarse en el caso de la Prehistoria del Ucayali central.

\section{Rio Napo, Ecuador}

La exploración del río Napo llevada a cabo por Evans y Meggers (1968) da como resultado una secuencia de cuatro fases donde la evidencia más abundante corresponde a la Fase Napo que pertenece a la subtradición Miracanguera. Los asentamientos de esta fase generalmente eran de forma lineal (Tabla 9) y tenían una dimensión modal mucho mayor a la de los túmulos 
de la Fase Marajoara, aunque el tamaño máximo de los asentamientos no rea del todo distinto si se combina las áreas de superficie de los fúmulos de 1-15. En N-P-2 se observó que las áreas de densa concentración de restos te alternaban con zonas esencialmente esteriles de basura cultural, probable. mente esto refleje la distribución de las casas en el asentamiento.

En contraste con los asentamientos de la Fase Napo, aquellos de las fases Yasuni Temprana y Tivacundo eran mucho más pequeños y claramente circulares. El único asentamiento habitacional de la Fase Cotacocha consistía de Hor áreas circulares de restos, cada una de alrededor de 5 metros de díámotro. Sin embargo, no existe suficiente información acerca de estas fases como para establecer generalizaciones; cuando se disponga de más datos decubriremos si los asentamientos lineales antecedieron a la aparición de la Fase Napo en esta región.

Tabla 9. Resumen de las Fases y Dimensiones de los Asentamientos en el rio Napo, Ecuador."

\begin{tabular}{|c|c|c|c|c|}
\hline$N-P=10$ & Yasuni & borde de colina & $18 \times 18 \mathrm{~m}$ & $254 \mathrm{~m}^{2}$ \\
\hline$N-P-7$ & Tivacundo & banco alto & $35 \times 30$ & 801 \\
\hline$N-P-1$ & Napo & banco alto & $650 \times 70$ & 45500 \\
\hline$N-P-2$ & Napo & banco alto & $500 \times 45$ & 22500 \\
\hline$N-P-3$ & Napo & banco alto & $500 \times 75$ & 37500 \\
\hline$N-P-4$ & Napo & banco alto & $\begin{array}{l}100 \times 20 \\
\text { (erosionado) }\end{array}$ & 2000 \\
\hline$N-P-5$ & Napo & banco & $75 \times 30$ & 2250 \\
\hline$N-P-6$ & Napo & banco alto & $150 \times 50$ & 7500 \\
\hline$N-P-9$ & Napo & banco alto & $30 \times 25$ & 572 \\
\hline$N-P-14 a$ & Cotacocha & banco glto & $5 \times 5$ & 20 \\
\hline$N-P-14 b$ & Cotacocha & banco alto & $5 \times 5$ & 20 \\
\hline
\end{tabular}

* Tomado de Evans y Meggers, 1968. 


\section{Rio Ucayali, Perú}

Desafortunadamente, la información acerea de patrones comunales dispo. nibles para los asentamientos del Ucayali central no es tan completa como la del río Napo, especialmente para los períodos tempranos que se encuentran a la base (o cerca de ella) de nuestras columnas estratigráficas. Incluso para los complejos posteriores, sólo puede determinarse con exactitud la distancia entre las excavaciones productivas. Igual que con el trabajo de Hilbert en el Amazonas central, este importante vacío en nuestra información puede atribuirse a la dificultad de lograr estimados precisos y rápidos de las dimensiones cuando el asentamiento está demasiado cubierto por una vegetación de bos. que tropical que ha llegado a su clímax; interrumpida solamente en asentamientos habitacionales aislados o campos de roce y quema. Al contrario de la experiencia de Evans y Meggers en el río Napo, no hemos hallado muchos asentamientos en la varzea cercana al curso actual del río (Lathrap, 1968) donde los inmigrantes mestizos han realizado una extensa tala. Más bien, nuestros asentamientos generalmente se han localizado en las antiguas tierras aluviales al borde de la varzea, donde sólo se ha limpiado pequeños trozos de terreno para cultivos de subsistencia. De todas maneras, se puede hacer unas cuantas observaciones útiles acerca de la dimensión y extensión de los asentamientos.

En primer lugar, podemos apreciar que ocupaciones con una extensión lineal mayor de 500 yardas se han identificado en los complejos Shakimo Tardío, Hupa-iya, Pacacocha, Cumancaya y Caimito (Tabla 10), con una distribución temporal que va desde aproximadamente 400 a.C. hasta cerca de 1400 d.C. Los complejos más tempranos son más conocidos en UCA 2 donde la forma de la colina en que se ubican no ha forzado ninguna forma particular de asentamiento. En contraste, UCA 17 toma todo el ancho de la colina, y TAM 2 -al igual que otros asentamientos Caimito en Imariacocha- está limitado en ancho y largo por la forma de la colina en que está ubicado. Las excavaciones de prueba para determinar la extensión de las diversas ocupaciones de UCA 2 constifuirán grandes aportes, como también las futuras pruebas en UCA 34 que bien podría ser el asentamiento más grande hasta ahora descubierto en el Ucayali central.

Unicamente en UCA 17 se han realizado pruebas para determinar la na. turaleza y extensión del asentamiento que pensamos sea demasiado pequeño para el Ucayali central, aunque es más grande que la mayoría de túmulos de la Fase Marajoara en la boca del Amazonas. Las excavaciones en UCA 17 consistieron en una zanja de 85 pies, que iba desde el costado de la colina hacia su centro, una zanja de 25 pies en la cima y dispersos por el lugar, siete cuadrados de 5 pies excavados hasta una profundidad de tres pulgadas. El análisis de los materiales recuperados en las tres primeras pulgadas de cada uno de los cuadrados de 5 pies que conformaban las zanjas y de los pozos de prueba, permitió segregar estas unidades en dos grupos diferenciados: unidades de alta densidad en las que se halló más de 50 tiestos y unidades de baja densidad en las que había menos de 20 tiestos. Entonces se observó 
Tabla 10. Tamaños y Dimensiones de los Asentamientos en el Ucayali Central.

$\begin{array}{lllll}\text { Asentamiento } & \text { Compiolo } & \text { Características } & \text { Dimensiones } & \text { Area } \\ \text { UCA } 2 & \text { Shakimu } & \text { morrolineal } & 600 \times-y d s . & - \\ \text { UCA } 2 & \text { Hupa-iya } & \text { morrolineal } & 600 \times- & - \\ \text { UCA } 34 & \text { Yarinacocha } & \text { morro } & 93^{*} \times 40^{*} & 3720 \text { yds.2 } \\ \text { UCA } 1 & \text { Paracocha } & \text { morrolineal } & 270 \times 70 & 18900 \\ \text { UCA } 2 & \text { Paracocha } & \text { morrolineal } & 600 * \times- & - \\ \text { UCA } 4 & \text { Paracocha } & \text { morrolineal } & 70 * \times 20 * & 1400 \\ \text { UCA } 10 & \begin{array}{l}\text { Cashibocaño } \\ \text { Nueva }\end{array} & \text { morrolineal } & 70 * \times 70 * & 3850 \\ \text { UCA } 17 & \text { Esperanza } & \text { morro } & 160 \times 100 & 16000 \\ \text { UCA } 10,33 & \text { Cumancaya } & \text { morrolineal } & 500^{*} \times- & - \\ \text { UCA } 22 & \text { Cumancaya } & \text { terraza antigua } & 500^{*} \times- & - \\ \text { TAM } 2 & \text { Caimito } & \text { morrolineal } & 600 \times 200 & 120000\end{array}$

* Distancias máximas entre excavaciones productivas.

que las unidades de alta densidad estaban concentradas en los bordes del asentamiento mientras las unidades de baja densidad lo estaban hacia el centro. En otras palabras, un óvalo de densa acumulación de basura rodea a una zona relativamente estéril en el centro. Incluso la estratigrafía natural de la zanja larga, demuestra que la profundidad de tierra laterizada bajo la superficie decrece a medida que la zanja se acerca al centro del asentamiento, lo cual sugiere que en esta zona la basura acumulada se había limpiado sistemáticamente.

Aunque desde un punto de vista cronológico se conoce bastante bien la historia cultural del Ucayali central, sabemos muy poco acerca de los patrones de asentamiento en la prehistoria de esta área. Sin embargo, por lo menos queda claro que los asentamientos relativamente grandes estuvieron presentes alrededor del 400 a.C. Si no eran lineales eran muy grandes, con una dimensión de treinta hectáreas o más - demasiado terreno y mucha basura-para la pequeña familia extensa que Steward considera característica de la montaña pervana. 


\section{PATRONES ETNOGRAFICOS DE ASENTAMIENTO}

A partir del análisis de la evidencia arqueológica aparecen por lo menon dos variantes principales de los patrones de asentamiento prehistóricos: II neales y no-lineales. Para dar cuenta del significado de esta distinción debemoi mirar hacia la evidencia etnográfica como lo hicieron Meggers y Evans (1957) en su pionera monografía acerca de la arqueología amazónica.

Quizás el tipo de asentamiento más común hoy en día en el bosque tropiejll sea la vivienda aislada unifamiliar del tipo descrito por Steward como ca racterística de la montaña. Además de los muchos mestizos que viven de esto modo, también lo hacen indígenas como los Campa (Varese, 1968) y muchal) Tikuna en franca imitación de los neo brasileños (Nimuendajú, 1952). Tipl camente, el asentamiento consistiría en un área circular de unos 30 metra de diámetro con una casa más o menos al centro. Si el piso de la casa estu. viera elevado debería existir un área de acumulación de basura bajo el piso, un área casi circular alrededor de la casa de donde el desecho se limpla sistemáticamente y un poco más lejos un área relativamente densa de acu. mulación de basura que se extraía del terreno inmediato a la casa.

La comunidad de casa única multifamiliar es más conocida en la zona amazónica del nor-oeste donde aquellas viviendas están ocupadas por tribus tales como los Witoto, los Jibaro y los Cubeo, y es la forma comunitaria tradicional de los Tikuna quienes recién han inmigrado a las orillas del Amazonas. Existen por lo menos dos variantes principales de estas casas multifamiliareat circulares y ovaladas. Los Witoto utilizaban ambas formas (Whitten, 1915, Farabee, 1922) seguramente según el tamaño del grupo. Una sola casa podla ser ocupada hasta por cien familias en igual cantidad de departamento individuales distribuidos en el perímetro de la viviendo cuyo centro se utllizaba para reuniones y danzas. Farabee vio una casa en construcción para veinte familias. Tenía 60 pies de largo, 45 de ancho y 30 de alto. Si tuviera que agrandarse para albergar a cien familias podría alargarse hasta unos 300 pies pero probablemente el ancho permanecería igual. Aparentemente, las aldeas tradicionales Witoto algunas veces consistían de varias de esas casa: (Steward, 1948c) dispuestas en círculo, según el informante de Farabee. Parece existir diversas variantes significativas de los interiores de estas casas largas? hablar de ellas estaría fuera del propósito de este trabajo. Incluso la estructura de la casa misma parece haber tenido un peso simbólico importante (Goldmang 1963; Reichel Dolmatoff, 1968).

En el tercer patrón de asentamiento más importante del bosque tropical las casas están sifuadas alrededor de una plaza abierta. Como señalábamos antes, aparentemente los Witoto utilizaban esta distribución igual que los Tupinambá del Período de Confacto (Métraux, 1948). Del mismo modo, la aldea Trumai del alto Xingú consistía de varias casas alrededor de una plaza (Murphy y Quain, 1955) como también las aldea's Kuikuru (Carneiro y Dole, 1956-57) y las Camayura (Oberg, 1952). Esta forma también es típica de los hablantes Ge del Timbira oriental (Nimuendajú, 1946) y los Apinayé (Ni. muendajú, 1939) del Mato Grosso quienes utilizan la galería forestal para 
lardines pero también salen de expedición durante medio año, actividad lleve a clasificarlos como tribus Marginales en el Handbook. De manera lor, los Mojo, hablantes Arawak del sigjo XVII, tenían de 50 a 400 casas inilbuldas en una plaza abierta con una casa de hombres al centro (Métraux, 1917). La forma de todas estas comunidades de tipo plaza central, deben abier nido más o menos circular pudiendo variar de famaño considerablemente auin la dimensiones de la plaza que no estaba necesariamente en relación litiecta al número de casas o personas en la camunidad. Podía, por ejemplo, hiaber dos círculos de casas alrededor de una plaza pequeña en lugar de 1 misina cantidad de casas alrededor de una plaza amplia. Otro factor que implica el panorama se refiere a la posibilidad de comparación entre las amunidades de pastos abiertos y las de bosque donde muchos árboles tendrían iumbarse con herramientas primitivas. Podríamos suponer que las plazas las comunidades de Bosque Tropical eran mucho más pequeñas que las pastos abiertos con el mismo número de habitantes. Aún así, el tamaño algunas comunidades actuales de tipo plaza central podría tomarse en uenta para la comparación con la evidencia arqueológica.

Ia comunidad Canella de Ponto tiene ese patrón de asentamiento circular. Ii plaza mide alrededor de 300 metros de diámetro, a su alrededor se disWibuyon treinta y un casas, cada una de las cuales alberga una familia exlena o quizá diez personas. Los Canella hacen un gran esfuerzo por mantener lis hreas de las casas y de la plaza libres de basura, aunque en realidad Ii unleo que se llega a mantener libre de vegetación durante todo el año ii la parte central de la plaza, los caminos radiales y el bulevar circular 1. lante de las casas. Cualquier desperdicio que caiga en estas áreas es arroindo fuera del anillo de casas (Nimuendajú, 1946). Por lo tanto, si un arqueb́logo ivviura que desenterrar Ponto luego de haber estado abandonado durante un $11 i m p o$, podría identificar el área de la plaza, incluyendo el bulevar delante to la casas, el anillo de casas, y el anillo de basura cultural fuera del anillo 16. casas. El asentamiento sería casi circular con un diámetro de unos 350 metros, un ărea aproximada de diez hectáreas.

Ia comunidad Camayura en Tuatuari tiene una distribución similar aunque ulgo más pequeña. La plaza mide sólo 100 yardas de diámetro al que el ancho de las casas añade otros 10 metros a cada lado. De este modo, el área liabltacional del asentamiento fendría unos 120 metros de diámetro con un unillo adicional de basura que podría tener otros 10 a 15 metros de ancho a cada lado dando al asentamiento un área total de 150 metros, cubriendo un poco más de una hectárea y media en la cual vivían 110 personas, Si el asontamiento hubiera eștado ocupado durante mucho tiempo, se podría disInguir por estar un tanto más alto que el resto del terreno debido al desperdicio alli depositado, el área habitacional estaría un poco rebajada por la constante limpieza realizada mientras el asentamiento estuvo ocupado.

Las aldeas Tupinambá pueden tener cuatro a ocho casas agrupadas allecledor de una plaza central y el área rotal en medio de una palizada. Aunque Métraux no da información acerca del tamaño total, sí indica que lan casas individuales tenian de 50 a 500 pies de largo por 30 a 50 de 
ancho. Una casa de tamaño normal tendría entre 250 y 300 pies de largo y albergaría a unas 30 familias, probablemente bastante más de 100 personas con algunas casas albergando a más de 200 individuos. Asi, una aldea Tupinambá normal podría tener una población entre 400 a 1,000 personas.

El cuarto patrón de asentamiento hoy en día en el bosque tropical es característico de grupos como los Piro y los Shipibo de la montaña peruana. La típica comunidad Shipiba consiste de una línea de casas extendidas a lo largo de una loma o terreno elevado, generalmente mirando hacia un lago o con menos frecuencia, hacia un rlo. Generalmente cada casa alberga a una familia nuclear con casas adyacentes casi siempre ocupadas por miembros de la familia extensa matrilocal. Frente a la línea de casas se encuentra una amplia plaza que las mujeres de la comunidad mantienen cuidadosamente libre de desecha y vegetación con ayuda de escoba y machete. Detrás de las casas hay una franja angosta que también se mantiene limpia. Las áreas de desecho están confinadas a las zonas de selva delante de la plaza y tras las casas. Ast el típico asentamiento Shipibo de sólo cuatro casas tendría aproximadamente 100 yardas de largo por 300 de ancho. A ambos lados del asentamiento habría un área de acumulación de desecho de quizá 5 a 7 yardas de ancho. Al interior de este óvalo de basura cultural el arqueólogo podría encontrar una zona estéril a menos que las casas fuvieran piso, lo cual es frecuente si la aldea no está muy por encima del nivel de inundación. Sin embargo una comunidad shipiba de cuatro casas generalmente sería Fequeña, 10 a 115 casas en línea sería lo más común. En este caso el largo del monticulo de basura sería mís bien de 250 a 375 yardas aunque el ancho permanecería más o menos igual. Las comunidades shipiba muy grandes tales como San Francisco de Yarinacocha. consisten de dos hileras de casas frente a frente con una amplia plaza al centro. Tal asentamiento podría tener entre 40 y 60 yardas de ancho por más de varios cientos de largo.

En 1956 Lathrap (1962) excayó un basural shipibo al frente de casas que hablan estado ocupadas durante unos treinticinco años. Encontró que la parte más alta del basural era de sólo 4 pulgadas mientras que la profundidad promedio tenla solamente 2 pulgadas - un ritmo de acumulación de sólo .06 a .12 pulgadas anvales- no mucho detrito para un asentamiento que ha sido ocupado durante mucho tiempo según los standards comúnmente aceptados para el bosque tropical.

La información acerca de los modernos patrones de asentamiento en el bosque tropical y el Mato Grosso está resumida en la Tabla 11. Probablemente las cifras estén en el orden correcto de magnitud pero debe aclararse que son sólo "adiviestimados". Sin embargo, revelan que las formas de asenta. miento lineales y no-lineales identificadas en el récord arqueológico se siguen manteniendo por los actuales habitantes del bosque tropical. La tabla también indica que esta división tan simple guarda una importante distinción en cada categaría. Las comunidades lineales incluyen tanto a las largas casas únicas comunales como las hileras de casas; las comunidades no-lineales incluyen a los asentamientos de casa unifamiliar y a las comunidades de plaza central. 
Tabla 11. Estimado de dimensiones, tamaños y población de algunas Comu. nidades modernas de la Amazonía.

Grupo

Campa

Witoto

Canela

Camayura

(casa de pequeña extensión)

(casa de grande extensión)
Dimensiones

30 yds. $x \quad 30 y d s$

55 yds. $\times 30$ yds.

1,650

120 yds. $\times 55$ yds.

6,600

350 yds. $\times 350$ yds. $\quad 96,250$

310

150 yds. $\times 150 y d s . \quad 17,678$

110

Shipibo

$$
\begin{aligned}
& \text { (pequeña) } \\
& \text { (mediana) } \\
& \text { (grande) }
\end{aligned}
$$

100 yds. $\times 30$ yds.

3,000

16

375 yds. $x \quad 30$ yds. $\quad 11,250$

60

$400 y d s . x \quad 60 y d s . \quad 24,000$

124 
Al arqueólogo le podría convenir asumir que la diversidad de patronef de asentamiento actuales es la misma que la del pasado, pero sería un infortunio que así fuera. Entonces el historiador de la cultura Amazónica no podría hacer otra cosa que dilucidar la distribución prehistórica y la evolv. ción de los tipos conocidos. No contribuiría en nada al conocimiento antropo lógico de la variación culfural humana en el bosque tropical. Afortunadamente. del récord etnohistórico sabemos que éste no es el caso. Tanto los Conibo como los Cocama tenían puęblos de grandes casas multifamiliares, con po blaciones que bordeaban los 2,000 individuos (Figueroa, 1904; Raimondil] 1876). No sabemos cuál era la distribución de estas aldeas, ni tampoco sabemos mucho acerca de su organización política. Estas. son interrogantes que el récord arqueológico resuelve con propiedad. Sabemos desde ya que no se adecúan al modelo de ninguna tribu moderna del bosque tropical. Más bien, fueron pioneras en el sentido Circum-Caribe.

\section{UNA COMPARACION ENTRE LOS PATRONES DE ASENTAMIENTOS ETNOGRAFICOS Y ARQUEOLOGICOS}

Los pequeños asentamientos casi circulares característicos de la Fase Ananałuba en el Marajó, caben perfectamente dentro de las dimensiones antes mencionadas para asentamientos de casa unifamiliar, pero la cantidad y profundidad de basura desdicen esta conclusión. Como sugieren Evans y Meggers, sería más lógico pensar en una pequeña casa multifamiliar. Parece que la comunidad Ananatuba consistió de una casa de ese tipo y excepcionalmente dos juntas como en J-7. Si así fuera, las comunidades de la Fase Ananatuba probablemente consistieron de 40 a 100 individuos.

En la Fase Mangueiras, los asentamientos habitacionales son un poco mayores, generalmente coinciden con los parámetros sugeridos para una pequeña casa multifamiliar como la que Farabee vio construyendo a los Witoto. Dos asentamientos probablemente de dos o tres de tales túmulos habitacionales y el asentamiento más grande de la Fase Mangueira posiblemente incluyeron tres a cuatro casas pequeñas ordenadas en una fila. En pocas palabras, el tamaño de la comunidad parece haber incrementado un poco, el más grande incluyendo de 150 a 200 habitantes.

Las comunidades de la Fase Formiga eran aparentemente casi del mismo lamaño; pero en algunos casos estaban construidas en túmulos artificiales, algunas veces dispuestos en un diseño definido en el asentamiento J-6. Cada uno de los dos túmulos más grandes de este asentamiento pudo haber soportado dos pequeñas casas Witoto, pero ninguno de los túmulos más pequeños es lo suficientemente grande como para haber aguantado aunque sea una. Quizá estaban destinados a alguna función especial como cocinar, elaborar cerámica o para reclusión femenina. En cualquier caso, no aumentarían demasiado la población de la aldea que debe haber sido aproximadamente la misma que la de una de las mayores comunidades de la Fase Mangueiras. 
El patrón de asentamientó de la Fase Maraloara se trasladó a los bancos de un pequeño arroyo al borde del cual se construyó túmulos artificiales. Alqunas comunidades de la Fase Marajoara eran casi del mismo tamaño que las de la fase anterior, pero ofras parecen haber sido mucho mas grandes. 3 asumimos que la erosión ha disminuido algunos de los fúmulos del asentamiento J-15, esta comunidad puede haber incluido alrededor de once casas del típico tamaño Tupinambá y vointidós do tamaño Witoto. El asentamiento J-1:4 puede haber contenido una casa de tamaño Tupinambé y dos tamaño Witoto; el asentamiento $J-22$ una casa de tamaño Tupinambá; y el asentamiento J-25 una de tamaño Witoto. De acuerdo a estas cifras, la población de la aldea mús grande debe haber sido de varios miles de personas mientras la más pequeña de cerca de 40 individuos. Si asumimos que el área de superficie de los túmulos era en su fotalidad un espacio babitacional, podemos aplicar la cifra de diez metros cuadrados por persona, dada por Narroll (1962) y LeBlanc (1970), para arribar a una suma de alrededor de 3,500 habitantes en al asentamiento $\mathrm{J}-15$, casi la misma que la derivada por el otro método.

Mientras que la organización sociopalítica de las tres primeras fases de Marajó bien podrla haber sido "tribal" en el sentido de Service (1969), la unidad política durante la Fase Marajoara debe haber sido una jefatura tribal en toda su dimensión. Como muchos autores reconocen, tal complejidad sociopolítica no es característica de la Cultura de Nivel de Bosque Tropical como caricaturizada anriba. Pero, zacaso es necesario dar cuenta de la aparición de tal complejidad por la rápida y directa inmigración de una fuente externa a la Cuenca Amazónica? A partir de la evidencia expuesta abajo, me parece que no lo es.

Desafortunadamente, los datos del Amazonas central no están bien definidos ni son tan fácilmente interpretables como los de Marajó. Sin embargo, son evidentes algunos hechos generales. Primero, los asentamientos son mucho más grandes que los de la Fase Marajoara. Los asentamientos lineales de la subtradición Guarita alcanzan longitudes hasta de 6,000 metros por 400 metros de ancho. El asentamiento más largo del cual se tienen pegistradas las dimensiones, cubría 80 hectáreas, en contraste con la superficie total del asentamiento $\mathrm{J}-15$ de la Fase Marajoara que cubría sólo unas tres hectáreas y media. Incluso asumiendo que el área de desecho de los túmulos de la Fase Marajoara fuera varias veces el tamaño de la superficie actual de aquéllos; la dimensión de los asentamientos de Fase Marajoara es de un orden de magnitud distinto que los de la subiradición Guarita en el Amazonas central. La evidencia actual sugiere que los asentamientos de la subtradición Miracanguera en el Amazonas central no fueron tan grandes como los de la subtradición Guarita pero, tal como ha sido indicado anteriormente, es muy posibla que la extensión actual del asentamiento ltacoatiara sea considerablemente menor de lo que fue en el pasado. Bien podría haber tenido mucho más de 80 hectóreas.

Ya que tenemos tan poca evidencia que incida directamente en el patrón de asentamiento en estos asentamientos, resulta extremadamente dificil hacer 
ningún tipo de estimados poblacionales. Sin embargo, como los asentamientos eran claramente lineales y la cerámica pertenece a la misma tradición ce. rámica que los asentamientos de la Fase Marajocira, parece razonable asumir que el patrón de asentamiento también era similar. El asentamiento Manaca. purú podría fácilmente haber acomodado 120 casas del tamaño promedio Tupinambá conteniendo una población proyectada de unas 18,000 personas - un número incréble- incluso un cuarto o un octavo de esta cifra sería demasiado para conformar el patrón predicho para el Nivel de Cultura de Bosque Tropical. Si aplicamos la razón shipiba del tamaño de asentamiento a la población $\left(200 \mathrm{~m}^{2}: 1\right.$ persona) obtenemos una proyección de población de alrededor de 4,000 habitantes para el asentamiento Manacapurú, más o menos la misma que la población del asentamiento J-15 de la Fase Marajoara. La verdadera población prehistórica del asentamiento Manacapurú probablemente fue algo mayor que esta cifra mínima ya que posiblemente la gente vivía en casas multifamiliares como las de los Tupinambá o Cocama, más que en casas de familia extensa caracteristicas de los Shipibo actuales.

Los asentamientos de la Tradición de Línea Fina Incisiva en el Amazonas central parecen haber sido ovalados y mucho más pequeños que los de la Tradición Policrama, pero los asentamientos de Santarem pueden haber sido del mismo tamaño. Ciertamente la presencia de terraplenes artificiales sugiere una organización política altamente desarrollada capaz de dirigir una tarea economicamente no productiva para quienes trabajaban en ella. En pocas palabras, la isla Marajo no fue el único lugar en la Cuenca Amazónica que iuvo una organización socio-política demasiado campleja para encajar en el modelo de Nivel de Cultura de Bosque Tropical.

Los asentamientos de la subtradición Miracanguera en los ríos Napo y Ucayali son también mucho más largos que anchos, coincidiendo con el patrón establecido en el Amazonas central y la boca del Amazonas. En cuanto al tamaño, los asentamientos del alto Amazonas concuerdan más con los de Marajoara que con los del Amazonas central. Si N-P-i consistió únicamente de una fila de casas tamaño Tupinambá, la pablación podría haber sido de mil doscientas a mil ochocientas personas. Utilizando el modelo shipibo obtenemos una población de doscientos veinticinco habitantes. Aunque este número ni siquiera se acerque a los estimados para el asentamiento Manacapurú, es de todas maneras difícil imaginar una organización social igua. litaria de la Cultura de Bosque Tropical inclusa para la Fase Napo. Una or. ganización política igualitaria es aun menos probable en el caso del Complejo Caimito en el Ucayali ya que la extensión del asentamiento es casi tres veces la de $N-P-1$. Si todas las colinas que rodean Imariacocha estuvieron habitadas por gente del complejo Caimito como sugiere Lathrap, deben haber sido muchos miles de personas los que vivieron alrededor del lago y participaron del sistema social. Con seguridad una sociedad dividida en rangos o incluso estratificada (Fried, 1967) es más probable que una estructura igualitaria en la Cultura de Bosque Tropical.

Permítasenos decir, sin embargo, que nadie piensa que la Tradición Polícroma pertenece al Nivel de Cultura de Bosque Tropical. Evans y Meggers 
(1968) la traen desde las montañas ecuatorianas o colombianas, pero el fe. chado de la Fase Napo, de la secuencia del Amazonas central y de la Fase Marajoara simplemente no respaldan este punto de vista. Por otro lado, Lathrap (1970) argumenta que la Tradición Polícroma se desprende de la Tradición Barrancoide, probablemente en el bajo y medio Amazonas. La información resumida en este informe tiende a apoyar-la opinión de Lathrap puesto que los asentamientos más grandes y mayores densidades de pobla. ción parecen estar en el Amazonas central. Sin embargo, el asunto sería mucho más sólido si se pudiera demostrar que los asentamientos del Amazonas central anteriores a la Tradición Policroma eran también mayores que los tamaños atribuibles al Nivel de Cultura de Bosque Tropical.

Como ha sido señalado anteriormente, lasentamiento Itacoatiara del Amazonas central era al parecer mucho mayor de lo que queda. El que por lo menos un tipo de cerámica 'de diagnóstico' Barrancoide se halle en ambas secciones del asentamiento sugiere que las dos estuvieron ocupadas con anterioridad a la subtradición Miracanguera. En consecuencia, debe asumirse que la extensión horizontal del basural Barraneoide es tan grande o mayor que el del amponente Miracanguera. El asentamiento debe haber sido enorme antes de la aparición del componente Miracanguera, de esto podemos dedueir que la aparición de la Tradición Policroma en el Amazonas central también estuvo precedida por grandes poblaciones y complejas organizaciones sociopolíticas.

En contraste, la llegada de la subtradición Miracanguera a la boca del Amazonas y al río Napo aparentemente no esfuvo precedida por grandes poblaciones. En el Ucayali la situación parece ser bastante diferente pese a que la información que pueda obtenerse para aportar sobre el problema es mucho menos amplia de lo que nos gusiaria.

Las excavaciones de Lathrap (1962) en UCA 2 demostraron que el desecho de los complejos superimpuestos Shakimu Tardío y Hupa-iya cubría una distancia de por lo menos $\mathbf{8 0 0}$ yardas. Si asúmimos un ancho mínimo de asentamiento, obtenemos un patrón de asentamiento de 'fila-única-de-casas'. Quizá podrian exfenderse en este corredor tres o cuatro casas tamaño Tupinambá y la población sería de 400 a 500 personas, posiblemente no muy grande para el Nivel de Cultura de Bosque Tropical, aunque Fried (1967: 113) sugiere que tales cifras son características de sociedades divididas en rangos. Pero cuatrocientas a quinientas personas es un estimado de tope minimo. Con toda probabilidad los asentamientos fueron bastante más grande de lo demostrado hasta ahora y la población posiblemente también mayor. ¿Podría este gran número de personas haber vivido juntos el tiempo suficiente como para depositar la cantidad de basura que dejaron si hubieran estado en el Nivel do Cultura de Bosque Tropical? Yo creo que no, y estamos hablando de fechas bastante anteriores a la Era Cristiana (Lathrap, 1970). En la Tradición Pacacocha se da el mismo tipo de problema aunque no de forma tan aguda. Los asentamientos más grandes conocidos de esta tradición sólo tienen de quinientas a seiscientas yardas de largo por un ancho desconocido, pero la capa de desecho tiene varias pulgadas de alto, lo cual sugiere una ocupación del 
asentamiento más bien prolongada si se recuerda que un basural shipibo promediaba sóló 2 pulgadas de profundidad después de treinticinco años de ocupación del lugar. En todo caso esto no refuerza el modelo de Steward sobré el patrón de asentamiento de montaña que caracteriza a las pequeñas familias extensas que se movilizan cada cierta cantidad de años. Parece enteramente factible que una sucesión de jefaturas tribales en el Ucayali precedió la llegada del Complejo Caimito por más de mil años.

\section{CONSIDERACIONES GENERALES}

En estas pocas páginas he dedicado un gran esfuerzo a la identificación de los probables patrones de asentamiento de verrias comunidades Amazónicas de la prehistoria, principalmente con el propósito de obtener algún estimado sobre el número de personas que vivian en ellas para así lograr una visión del nivel general de complejidad socio-política que los caracterizaba. El pa. trón de asentamiento tiene otras implicancias que merecen la atención del arqueólogo.

De lo que sabemos acerca de la etnografía y etnohistoria de la Cuenca Amazónica, la casa aislada unifamiliar indica lazos sociales extremadamente difusos con otros segmentos de la sociedad. Tal patrón podría no haber sido viable en las condiciones de guerra constante que caracterizaba a los ríos grandes de la Amazonía desde antes de Cristo. Este patrón puede haber tenido éxito en regiones remotas, pero la continua belicosidad de las tribus interfluviales desde las épocas más tempranas registradas hasta el presente hace de esta posibilidad algo bastante improbable. En todo caso, la casa aislada unifamiliar parece ser producto de la pax iberica, posiblemente sólo bajo condiciones de baja densidad poblacional surgida con la introducción de enfermedades europeas.

Actualmente la 'casa aislada multifamiliar es característica del Amazonas noroccidental, pero la evidencia de Marajó sugiere que estuvo más extendida en el pasado. Donde se la encuentre, es indicativa de una sociedad en pequeña escala organizada según líneas de parentesco, pero no podría haber sobre. vivido a la presión de los bravos guerreros de las sociedades de gran escala. Incluso en siglo XX grupos como los Jíbaro (Stirling, 1938) y los Witoto (Whiffen, 1915) hallaban prudente -por razones de defensa- ubicar sus casas lejos de los ríos principales.

Las sociedades de gran escala, como las que habitaban los ríos grandes de la Amazonía al tiempo del Contacto, pueden haber vivido en comunidades ya sea del tipo de plaza central o lineal larga. La primera puede haber sido útil como medida de defensa ya que tiene la virtud de presentar el perímetro de defensa más pequeño para un área dada. Ya que sus aldeas estaban situadas en palizadas, por lo menos los Tupinambá estaban al tanto de este problema. Sin embargo, no debe pasarse por alto el que este patrón de asentamiento esté firmemente asociado a una visión particular del mundo (Levi-Strauss, 1963). Que grupos dispersos tal como los Ge-Bororo, los pobla- 
dores de las Islas Trobriand (Malinowski, 1929) y los Murgnin (Warner 1937) mantuvieran este patrón sin otras medidas de defensa significativas indica que las consideraciones simbólicas pueden haber sido importantes para la olección de este patrón de asentamiento. Por otro lado, no existe evidencia para indicar que la selección del patrón lineal estuviera gobernada por principios simbólicos; las consideraciones económicas parecen tener mayor peso. Como hemos observado repetidamente los estudiosos de la Cultura de Bosque Tropical, la efectiva explotación del río es un rasgo fundamental de las Cuf. turas de Bosque Tropical. Por lo tanto el acceso al río probablemente fue el factor determinante. Más aún, que áreas realmente amplias de terrenos elevados no fueran disponibles excepto en los malecones y morros lineales, puede haber sido la causa de un cambio a este patrón de asentamiento de otro anterior que podía haber sido preferible simbólicamente, Si el simbolismo de un parrón de asentamiento circular fue importante podría haberse mantenido en la porción central del asentamiento mientras que el crecimiento suburbano desparramado caracterizaría a las áreas periféricas. No está demás observar que el centro de muchos pueblos y ciudades hispano-americanas preservan la asociación simbólica de iglesia y edificios gubernamentales frente a una plaza central mientras el resto del pueblo "crece a la buena de Dios".2 Cierłamente, es posible que un patrón similar de área central planificada y expansión periférica no planificada haya existido en los pueblos Amazónicos prehistóricos.

\section{CONCLUSION}

Con la evidencia disponible en la actualidad, ha sido posible obtener algunos estimados sobre el desarrollo de la complejidad socio-política en la Cuenca Amazónica. En la boca del Amazonas - de donde se dispone de la mejor información - el récord arqueológico empieza con la Fase Ananatuba que data aproximadamente de 1000 a.C. (PRONAPA, 1970). En aquella época el patrón de asentamiento característico era una sola pequeña casa larga que albergaba a unas 40 personas. Como variante excepcional, dos de tales casas podrian estar ubicadas muy juntas, dando a la comunidad una población total de unas 70 a 100 personas. En las siguientes fases: Mangueiras y Formiga, las casas pequeñas de ese tipo generalmente se encuentran en grupos de dos a cuatro casas para una población total de unas trescientas personas. Con la Fase Marajoara - alrededor de 500 d.C.- se da un rápido incremento en el número de habitantes que podían vivir en una sola comunidad, y también un nítido cambio en el patrón de asentamiento. Los túmulos artificiales que se habían construido primero en la fase formiga se extendían ahora hacia los bancos de un pequeño arroyo situando los túmulos más grandes hacia la boca. La población del mayor asentamiento debe haber sido de varios miles de personas. Debe haberse dado un combio concomitante en la organización socio-política, del nivel Tribal al nivel de Jefaturas Tribales según los términos de la clasificación Service (1962). La conclusión más obvia es que en ese tiempo hubo intrusión de una nueva eultura, pero la continuidad cerámica y la continuación de los túmulos artificiales sugieren la posibilidad de una explicación más sutil.

2 La expresión en inglés es "grows tike Topsy". 
Aunque el récord del río Napo no es tan completo, probablemente se dieren similares patrones de pablación y erecimiento político. En vista que los asenta. mientos de la Fase Yasuni parecen ser simples remanentes de asentamientos mucho mayores en la época en que fueron ocupados, no existe una base confiable sobre la cual estimar la población y la complejidad socio-cultural. La Fase Tivacundo que data aproximadamente de 500 d.C. (Evans y Meggers, 1968), parece haber tenido asentamientos casi del mismo tamaño que los de la Fase Ananałuba en Marajó - también queda indicada- una pequeña comunidad de casa larga si se toma como característica al único asentamiento cuyas dimensiones se conocen. Con la Fase Napo, que data de alrededor de 1,500 d.C. (Evans y Meggers, 1968) se da un vuelco a grandes asentamientos lineales parecidos a los de la Fase Marajoara en Marajó. No puede haber habido menos de 200 residentes en el asentamiento más grande de la Fase Napo y la población real puede haber sido mucho mayor. Nuevamente nos encontramos con una aguda variación demográfica cuando se introduce la cerámica de la subtradicíón Miracanguera.

En otras dos áreas exploradas existe un patrón bastante distinto. Aunque el récord arqueológico del Amazonas central llega sólo hasta Cristo, existe evidencia de asentamientos muy grandes, habitados por varios miles de individuos, que ya existían en esta temprana fecha. Podemos presumir, con bastante acierto, que existía una organización politica piramidal. Aunque la tradición cerámica varió con el tiempo, los grandes asentamientos siguieron siendo característicos $y$, con toda seguridad la organización política compleja también continuó. Ciertamente Orellana se topó con tales sociedades en 1542.

La secuencia arqueológica de la Cuenca Amazónica más larga y mejor do. cumentada es la del Ucayali central en el Perú Oriental. Desafortunadamente, los datos sobre el patrón de asentamiento están lejos de ser útiles. Sin embargo, la evidencia fragmentada que existe sugiere que las comunidades relativamente grandes han sido características de esta área por lo menos desde el Shakimu Tardío, alrededor de 400 a.C. (Lathrap, 1970) y continuaron siéndolo hasta el periodo histórico temprano a través de varios cambios en la tradición cultural. Cuando se introdujo la subtradición Miracanguera bajo el tinte del Complejo Caimito era simplemente la más tardía y la última de una larga historia de avanzadas organizaciones socio-políticas de río grande en la montaña peruana.

De este resumen de la evidencia queda bastante elaro que las grandes comunidades con organizaciones socio-políticas complejas no se desarrollaron ni en el río Napo ni en la isla Marajó. Evans y Meggers han dado cuenta de la súbita aparición de tales sociedades postulando una migración de los Andes del Norte hacia el Bosque Tropical. Como se ha visto, no es necesario recurrir a fuentes externas a la Cuenca Amazónica para explicar la existencia de sociedades complejas en estas áreas periféricas. Más aún, las fechas de rediocarbono indican una migración justamente en dirección opuesta. Las gran. des sociedades son incluso más antiguas en el bajo y medio Amazonas lo cual sugiere que el origen más probable de la subtradición Miracanguera estuvo tanto en el río Napo como en la boca del Amazonas. Si tales sociedades 
fueron o no fruto de un desarrollo indígena en ef Amazonas central, sólo lo dirá la investigación futura. También debemos considerar el hecho que sosiedades complejas relativamente grandes parezcan incluso más antiguas en el Ucayali central que en el medio Amazonas. ¿Podría considerarse al Ucayali como cuna de las culturas complejas? No lo creo. El desarrollo cerámico en el Ucayali estaba demasiado ligado al que tenía lugar en el Amazonas y más al norte, situando al Ueayali en una posición periférica. Mientras es posible que se hayan dado innovaciones políticas en el Ucayali, la precoz expansión de las tradiciones cerámicas sugiere que eran de un pueblo que habla desa. rrollado su sentido político mucho antes de ingresar al Ucayali. Quizá en última instancia debamos buscar los orígenes de las sociedades complejas fuera del bosque tropical, pero antes de enfrentarnos a tal necesidad, debemos aprender acerca de la historia de la Cultura de Bosque Tropical.

\section{Agradecimiento:}

Muchas de las ideas vertidas en este trabajo fueron expresadas originalmente en un esfuerzo conjunto con Donald W. Lathrap, y presentadas al $63^{\circ}$ Encuentro Anual de la Asociación de Antropología Americanista en Noviembre de 1964. Sin embargo Lathrap no intervino en la versión actual; por lo tanto no es responsable de ningún fragmento de su contenido. También deseo agradecer a Wesley R. Hurt por algunas sugerencias importantes que se incorporaron en la copia final. 
BATES, Henry Walter, 1864. The Naturalist on the River Amazons. John Murray, London.

CARNEIRO, Robert L. and GERTRUDE, Dole, 1956-57. La Cultura de los Indios Kuikurus del Brasil Central. Runa, Vol. 8, Ni 2, pp. 169-202. Buenos Aires.

EVANS, Clifford and BETTY, Meggers, 1968. Archeological Investigations on the Rio Napo, Eastern Ecuador. Smithsonian Contributions to Anthropology, Vol. 6. Wa. shington, D. C.

FARABEE, William Curtis, 1922. Indian Tribes of Eastern Peru. Papers of the Peabody Museum of American Archaoology and Ethnology, Vol. 10. Cambridge.

FIGUEROA, Francisco de. 1904. Relación de las Misiones de la Compañía de Jesús en el País de los Maynas. V. Suárez, Madrid.

FRIED, Morton H. 1967: The Evolution of Polilical Society. Random House, New York.

HARRIS, Joanne M. 1967. The Ceramic Se. quence at Cushillococha. Ms. Master's thesis, Unviersity of Illinois, Urbana.

HILBERT, Peter Paul. 1968. Archäologische untersuchungen am Mittleren Amazonas. Studien zur Volkerkunde. Band 9, Berlín.

LATHRAP, Donald W. 1962. Yarinacocha: Stratigraphic Excavations in the Peruvian Montaña. Ms. Doctoral dissertation, Mar. vard University, Cambridge.

1968. Aboriginal Occupation and Changes in the River Channel on the Central Ucayali, Peru. American Antiquity, Vol. 33, N9 1, pp. 62-79. Salt Lake City.

-1970. The Upper Amazbn. Praeger Pu. blishers, New York.

LEBLANC, Sfeven. 1970. An Addition to $\mathrm{Na}$ roll's Suggested Floor Area and Settlement Population Relationship. American Antiquity, Vol. 36, No 2, pp, 210-211. Salt Lake Cify.

LEVI.STRAUSS, Claude, 1963. Do Dual Organization Exist? Sfuctural Anthrepology, pp. 132-166. Basic Books, Ine., Now York.
LOWIE, Robert H. 1948. The Tropical Forestll An Introduction. En Julian H. Steward, Editor, Handbook of South American in. dians. Bureau of American Ethnloogy Bul. letin N: 143, Vol. 3, pp. 1-55. Washing. ton, D.C.

IM THURN, Sir Everard Ferdinand. 1883. Among the Indians of Guiana: Being Sketches Chiefly Anhtrepologic from the Interior of British Guiana. K. Paul, Treneb \& Con, London.

MALINOWSKI, Bronislaw, 1929. The Sexual Life of Savages. Harcourt, Brace \& World, Inc., New' York.

MEGGERS, Betty J. and CLIFFORD, Evans. 1957. Archeological Investigations at the Mouth of the Amazon, Bureau of American Ethnonology Bulletin N9 167. Washington, D.C.

METRAUX, Alfred. 1942. The Native Tribes of Eastern Bolivia and Western Matto Grosso. Bureau of American Ethnology Bulletin No 134. Washington, D.C.

1948. The Tupinamba. En Julian H. Ste. ward, Editor, Handbook of South American Indians. Bureau of American Ethnology Bulletin No 143, Vol. 3, pp. 95.134. Washington, D.C.

MURPHY, Robert F. and Buell Quain, 1955 The Trumai Indians of Central Brazil. Mono. graphs of the American Ethnological Socioty 24. Seattle.

MYERS, Thomas P. 1970. The Late Prehistoric Period at Yarinacocha, Peru. Ms. Doctoral Dissertation, University of Illinois, Urbana.

NAROLL, Raul. 1962. Floor Area and Seftle. ment Population. American Antiquity, Vol. 27. No 4, pp. 587-589. Salt Lake Cify.

NIMUENADAJU, Curt, 1939. The Apinoyé. Catholic University Anthropological Papers N: 8. Washington, D.C.

1946. The Eastern Timbira. Universily of California Publications in American Archaeology and Ethnology, Vol. XLI. Berkeley.

1952. The Tukuna. Universily of California Publications in American Archaeology and Ethnology, Vol. XIV. Berkeley, 
OBERG, Katervo. 1952. Indian Tribes of Nor. thern Mato Grosso, Brazil. Smithsonian Institution, Institute of Social Anthropolo. gy, Publication No 15. Washington, D.C.

PALMATARY, Helen C. 1960. The Archaeology of the Lower Tapajós Valley, Brazil. Transactions of the American Philosophical 5ociety, n.s., Vol. 50, Part 3. Philadelphia.

PRONAPA, 1970. Brazilian Archaeology in 1968: An Interim Report on the National Program of Archaeological Research. American Antiquity, Vol. 35, No 1, pp. 123... Salt Lake City.

RAIMONDI, Antonio. 1876. Historia de la Geografía del Perú. El Perú, Vol. II. So. ciedad Goográfica de Lima. Lima.

REICHEL-DOLMATOFF, Gerardo. 1968. Desana: Simbolismo do los Indios Tukano del Vau. pós. Universidad de los Andes y Editorial Revista Colombiana, Ltda. Bogotá.

SERVICE, Elman R. 1962. Primitive Social Organization. Random House, New York.

SIMOES, Mario F. 1967. Resultados Preliminares de una prospeccão arqueologica na regiao dos rios Gisoapi a Camara (Itha de Marajo). Atas do Simposio Sobre a Biota Amazonica, Vol. 2, pp. 207-224. Belem.

- 1969. The Castanheira. Site: New Evi. dence on the Antiquity and History of the Ananatuba Phase (Marajo lsland, Brazil). American Antiquity, Vol. 34, Ni 4, pp, 402-470. Salt Lake City.

STERNBERG, Hilgard O'Reilly. 1960. Radiocorbon Dating as Applied to a Problem of Amazonian Morphology. Comples Rendus du XVIII Congres Internacional de Geogre. phio, Vol. 2, pp. 322-424. Paris.
STEWARD, Julian H, 1948a. Tribes of the Montaña and Bolivian East Andes: An Introduction. En Julian H. Steward, Editor, Handbook of South American Indians. Bureau of American Ethnology Bulletin Ne 143, Vol. 3, pp. 507-533. Washing. ton, D.C.

1948b.-Culture Areas of the Tropical Forest. En Julian H. Steward, Editor, Handbook of South American Indians. Bureau of American Ethnology Bulletin No 143, Vol. 3, pp. 883.905. Washington, D.C.

Y948c.-The Witotoan Tribes, En Julian $H$. Steward, Editor, Handbook of South American Indians. Bureas of American Ethnology Bulletin No 143, Vol. 3, pp. 749-762. Washington, D.C.

STEWARD, Julian $H$. and Louis C. Faron. 1959. Native Peoples of South America. Mc Graw Hill, New York.

STIRLING, Mathew W. 1938. Historical and Ethnographical Material on the Jivaro In. dians. Bureau of Amarican Ethnology Bulletin Ne 117. Washington, D.C.

VARESE, Stefano, 1968. La Sal de los Carros: Notas Einográficas Históricas sobre los Campa de la Sélva del Perú. Universidad Peruana de Ciencias y Tecnologla. Departamento de Publicaciones Antropolegía. Lima.

WARNER, W. Lloyd, 1937. A Black Civilization: A Social Study of an Australian Tribe. Harper \& Row Publishers, Inc., Now York.

WHIFFEN, Thomas W. 1915. The Northwest Amazons: Notes on Some Months Spent among Cannibal Tribes. Constable and Company, Ltd,, London. 